\title{
Experimental Study of Falling Water Limita- tion under a Counter-Current Flow in a Vertical Rectangular Channel (1st Report, Effect of Flow Channel Configuration and Introduction of CCFL Correlation)*
}

\author{
Yukio SUDO**, Tohru USU1*** \\ and Masanori KAMINAGA**
}

\begin{abstract}
Counter-current-flow limitation (CCFL) experiments were carried out for both vertical rectangular channels and vertical circular tubes varying in size and in configuration of their cross sections to clarify CCFL characteristics in the vertical rectangular channels. Quantitative understanding of critical heat flux $(\mathrm{CHF})$ in a narrow vertical rectangular channel is required for the thermohydraulic design and safety analysis of research nuclear reactors in which flat-plate-type fuel is employed. Critical heat flux under downward low velocity is closely related to falling water limitation under a counter-current flow. Experimental results showed that the equivalent hydraulic diameter de, i.e., the width, and gap of the channel play an important role in determining the CCFL characteristics of a rectangular channel. However, a significant influence of channel length on CCFL characteristics was not observed in the ranges investigated. Using new dimensionless parameters, the authors propose a correlation for predicting the relationship between upward air velocity and downward water velocity based on the present experimental results.
\end{abstract}

Key Words: Critical Heat Flux, Counter-Current-Flow Limitation, Flat-Plate Type Fuel. Nuclear Reactor, Vertical Rectangular Channel, Vertical Circular Tube

\section{Introduction}

The understanding of critical heat flux (CHF) in a narrow vertical rectangular channel is essential for core thermohydraulic design and safety analysis of research nuclear reactors in which flat-plate-type fuel is employed. It has been reported that the $\mathrm{CHF}$, under such conditions as low downward velocity including the stagnant condition which would occur in anticipated operational transients and accidents, is closely related to the counter-current-flow limitation $(\mathrm{CCFL})^{(1),(2)}$, and many experiments and investigations on the CCFL have been carried out.

Wallis proposed a correlation of downward water

* Received 21st August, 1990. Paper No.88-1265B

** Japan Atomic Energy Research Institute. Tokaimura, Naka-gun, Ibaraki-ken 319-11, Japan

*** NKK Corporation, 1-1, Minamiwatarida-cho, Kawasaki-ku, Kawasaki 210, Japan velocity vs upward gas velocity on the CCFL in vertical circular tubes as shown below ${ }^{(3)}$ :

$$
\begin{aligned}
& \sqrt{j_{g d}^{*}}+m \sqrt{j_{L_{d}}^{*}}=C \\
& j_{g d}^{*}=j_{g d} \sqrt{\frac{\rho_{g}}{g d\left(\rho_{L}-\rho_{g}\right)}} \\
& j_{L d}^{*}=j_{L d} \sqrt{\frac{\rho_{L}}{g d\left(\rho_{L}-\rho_{g}\right)}}
\end{aligned}
$$

where $m$ and $C$ are constants. The $m$ is normally unity and the $C$ is 0.725 to 0.75 for a sharp-edged tube $^{(3),(4)}$ and 0.875 for a round-edged tube ${ }^{(3)}$.

Richter proposed a correlation of downward water velocity vs upward gas velocity shown below, based on a theoretical analysis on CCFL for both circular and annular tubes ${ }^{(5)}$ :

$$
\begin{aligned}
& \left(\frac{C_{w}}{4}\right) B o^{3} j_{\theta d}^{*} j_{L d}^{*}+C w B o j_{g d}^{*} \\
& \quad+150 C_{w j_{\theta d}}^{*}=1
\end{aligned}
$$

(for circular tubes)

$$
C_{w} B o^{3} j_{g w}^{*}\left(\frac{b}{W}\right) j_{L w}^{*}{ }^{2}+C_{w} B o j_{g w}^{*}
$$




$$
+150 C_{w} j_{\theta w}^{*} 2\left(\frac{b}{W}\right)=1
$$

(for annular tubes), where the average circumferential length $W$ is adopt ed for the representative length used in the $j_{z w}^{*}$ and $j_{L w}^{*}$ in the case of annular tubes.

Mishima carried out the CCFL experiments for the vertical rectangular channels and investigated the effect of the size of the channel gap, $b$ ( $b$; the shorterside length of the rectangular cross section) ${ }^{(2)}$. His experiments revealed that Eq. ( 1 ) could be applicable to the experimental results by adopting twice the channel width, $2 a$ ( $a$; the longer-side length of the rectangular cross section) for the representative length and 0.6 for the $C$ in Eq. ( 1 ).

On the CCFL of emergency core cooling water injected into the PWR downcomer, Sudo investigated the constants $m$ and $C$ in Eq. ( 1 ) based on the results of existing experiments for annular channels and correlated the $m$ and $C$ with the aspect ratio $b / W^{(6)}$.

Sudo and Ohnuki carried out the CCFL experiments for circular tubes, investigating the effect of $L / d$ ( $L$; channel length, $d$; channel diameter) on mass velocity of water falling through the channel against the upward gas mass velocity, and revealed that the mechanism of CCFL would be different between the case of $L / d>2$ and the case of $L / d \leqq 2^{(7)}$.

From the previous results mentioned above, it is considered that Eq. (1) is available for expressing the relationship of downward water velocity vs upward gas velocity in vertical rectangular channels. How. ever, it has not been made clear what effects the differences in the geometrical condition of the channel (i.e., configuration of cross section, such as circular tube, annular tube and rectangular channel) have on the constants $m$ and $C$ in Eq. (1).

In this study, therefore, CCFL experiments were carried out for the condition of $L / d e$ (de : equivalent hydraulic diameter of rectangular channel) $>2$ by changing the channel width $a$, the channel gap $b$ and the channel length $L$ in order to investigate the effect of these dimensions on the relationship of downward water velocity vs upward gas velocity. CCFL experiments were also carried out for circular tubes for investigating the differences in the characteristics of CCFL between the circular tubes and rectangular channels in this study.

This paper presents experimental results thus obtained on the CCFL characteristics for the configuration of rectangular channels.

\section{Nomenclature}

$a$ : Channel width (longer-side length of rectangular cross section) (m) $b$ : Channel gap (shorter-side length of rectangular cross section) (m)

$B_{o}:$ Bond number

$B_{o}{ }^{*}$ : Modified Bond number defined by Eq. ( 6 )

$C:$ Constant defined in Eq. (1)

$C_{w}:$ Wall friction factor

$d$ : Tube diameter $(\mathrm{m})$

de : Equivalent hydraulic diameter (m)

$\mathrm{g}:$ Acceleration of gravity $\left(\mathrm{m} / \mathrm{s}^{2}\right)$

$j$ : Superficial velocity $(\mathrm{m} / \mathrm{s})$

$j^{*}$ : Dimensionless superficial velocity

$L$ : Channel length (m)

$W:$ Circumferential length $(\mathrm{m})$

$\rho:$ Density $\left(\mathrm{kg} / \mathrm{m}^{3}\right)$

$\sigma:$ Surface tension $(\mathrm{N} / \mathrm{m})$

Subscripts
$b$ : Based on channel gap size
$d$ : Based on tube diameter
$g:$ Gas phase
$L:$ Water phase
$W:$ Based on circumferential length

\section{Experimental Apparatus and Method}

A schematic diagram of the experimental apparatus for CCFL is shown in Fig. 1. The test section is composed of an upper plenum, a lower plenum and a test channel made of lucite. Air supplied by a compressor flows upwards through the test channel and is exhausted from the exit of the upper plenum into the atmosphere, while part of the water supplied continuously into the upper plenum flows downwards through the test channel and the rest of the water overflows from the upper plenum with a constant water level of $550 \mathrm{~mm}$ kept in the upper plenum.

The downward superficial water velocity in the test channel was calculated with a rising velocity of water level in the lower plenum. The upward superficial air velocity in the test channel was measured with rotor flow meters, correcting the effects of

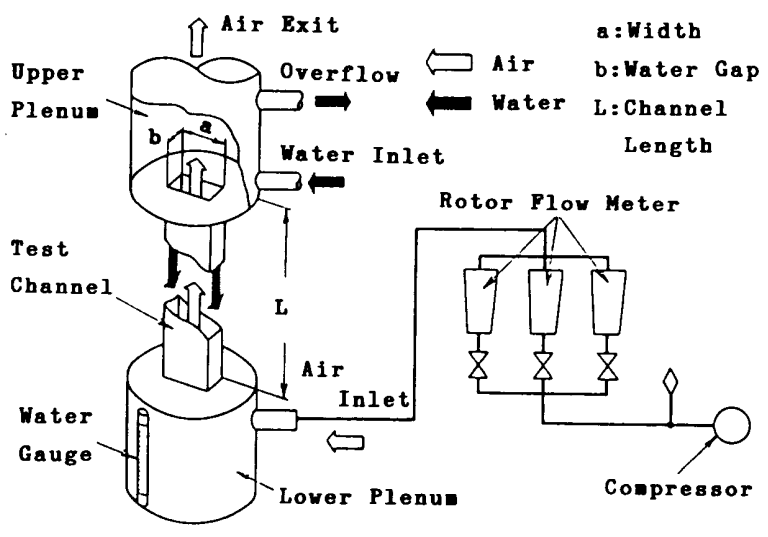

Fig. 1 Schematic diagram of test section 
fluid temperature and pressure.

The configuration of test channels and the experimental conditions investigated in this study are listed in Table 1.

\section{Experimental Results}

3.1 Downward water velocity vs upward gas velocity in circular tubes

Figure 2 shows the experimental results obtained for circular tubes in this study on the relationship of downward water velocity vs upward gas velocity. In the figure, the $\sqrt{j_{g d}^{*}}$ is adopted for the ordinate and the $\sqrt{j t_{d}^{*}}$ for the abscissa in accordance with the expression of Wallis ${ }^{(3)}$, taking $d$ and $L$ as parameters. The subscript $d$ in the dimensionless parameters, $j_{g d}^{*}$ and $j{ }^{*}{ }_{d}$ indicates that the tube diameter $d$ is adopted for the representative length in $j_{g}^{*}$ and $j_{k}^{*}$. Figure 2 demonstrates clearly that the experimental results for circular tubes are well represented by Eq. ( 1 ) with $C=0.7$, with no effects due to variations of the magnitude in $d$ and $L$, which were investigated in this study.

As already described, it has been reported that

Table 1 Configuration of test channels and experimental conditions investigated in this study

\begin{tabular}{|c|c|c|}
\hline \multirow[t]{2}{*}{ Tube } & 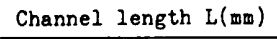 & 250,750 \\
\hline & Inner diameter $d(\mathrm{~mm})$ & $8,12,20$ \\
\hline \multirow[b]{2}{*}{$\begin{array}{l}\text { Rectangular } \\
\text { channel }\end{array}$} & Channel length $\mathrm{L}(\mathrm{mm})$ & $72,362,782$ \\
\hline & $\begin{array}{l}\text { Dimension of cross } \\
\text { section } \quad(\mathrm{mm}) \\
\text { [Width a } \times \text { Gap b] }\end{array}$ & $\begin{array}{l}66 \times 2.3,66 \times 5.3, \\
66 \times 8.3,66 \times 12.3, \\
33 \times 2.3,33 \times 5.3, \\
33 \times 8.3,33 \times 12.3\end{array}$ \\
\hline Fluid used $i$ & $n$ this experiment & water and air \\
\hline Temperature & $(\mathrm{K})$ & $288 \sim 300$ \\
\hline Pressure & $(a t m)$ & about 1 \\
\hline Superficial & air velocity & $1 \sim 17$ \\
\hline Water level & of upper plenum $(\mathrm{mm})$ & 550 \\
\hline
\end{tabular}

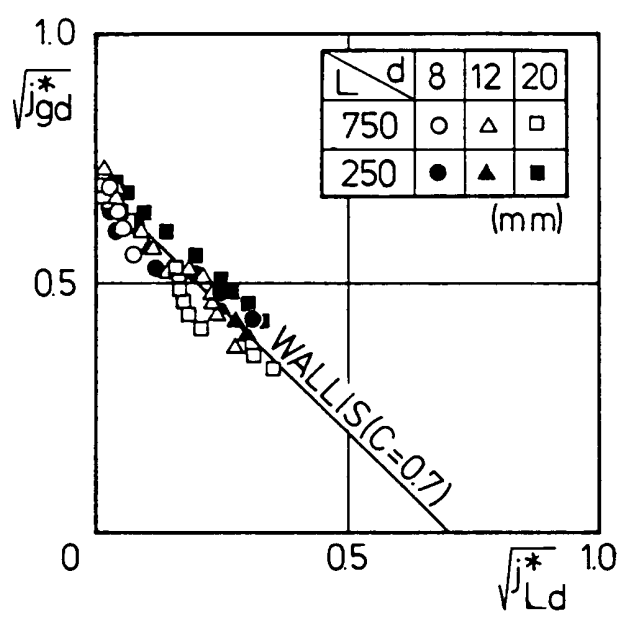

Fig. 2 CCFL experimental results for circular tubes the constant $C$ in Eq. ( 1 ) depends on the shape of the test channel end and in such cases as sharp-edged tubes investigated in this experiment, the value of 0.725 to 0.75 has been recommended for $C^{(3),(4)}$. It is, therefore, considered that the experimental results shown in Fig. 2 are well consistent with those of Wallis $^{(3)}$.

\section{2 Downward water velocity vs upward gas velocity in rectangular channels}

Figures 3(a) and 3(b) show the experimental results obtained for rectangular channels in this study on the relationship of downward water velocity vs upward gas velocity. In the figures, $\sqrt{j_{o b}^{*}}$ is adopted for the ordinate and the $\sqrt{j k_{b}}$ for the abscissa, taking $b$ and $L$ as experimental parameters. The subscript $b$ in $j_{a b}^{*}$ and $j_{b b}^{*}$ indicates the channel gap, $b$ is adopted for the representative length in Eqs. (2) and ( 3 ) instead of tube diameter, $d$. Figure 3 (a) shows the experimental results for $a=66 \mathrm{~mm}$ and Fig. 3(b) for $a=33$ $\mathrm{mm}$.

As seen clearly in Figs. 3(a) and 3(b), the smaller channel gap $b$ gives the larger dimensionless downward water velocity $j_{L_{b}}^{*}$ under the condition of a constant dimensionless upward gas velocity $j_{\theta b}^{*}$ and the smaller $j_{b b}^{*}$ gives the larger effect of channel gap $b$ on $j *_{b}$ in both cases of $a=33$ and $66 \mathrm{~mm}$. The effect of the magnitude in $L$ on $j_{L b}^{*}$ is not significant in the figures.

In Figs. 3(a) and 3(b) are illustrated the values of $m_{b}$ and $C_{b}$ which are recommended for representing the tendencies of the experimental data for each test channel configuration, that is, each combination of $a, b$ and $L$ in the rectangular channel when Eq. (1) is applied to the experimental data. The subscript $b$ in $m_{b}$ and $C_{b}$ indicates that the channel gap $b$ is adopted for the representative length in $j_{a}^{*}$ and $j_{L}^{*}$ when Eq. (1) is applied.

The following considerations have been taken into account in determining the values of $m_{b}$ and $C_{b}$ in Eq. ( 1 ) for each combination of $a, b$ and $L$.

i) At first, the effect of magnitude in $L$ on $j_{L b}^{*}$ was neglected because the effect was not significant, as seen in Figs. 3(a) and 3(b). Accordingly, the experimental data which are different only in the magnitude of $L$ can be represented by a single straight line.

ii) Next, the experimental data only with rather small $j_{g b}^{*}$ and rather large $j_{b b}^{*}$ were focused on in the application of Eq. (1) to the experimental data because the effects of $a$ and $b$ on $j_{b}^{*}$ are significant only for rather small $j_{\theta b}^{*}$ and therefore, the experimental data with $\sqrt{j_{a b}^{*}}$ larger than about 1.2 deviate significantly from each straight line recommended for each combination of $a$ and $b$ in Figs. 3(a) and 3(b). 
The following tendencies can be pointed out as remarkable features of the $C_{b}$ and $m_{b}$ thus determined in Figs. 3(a) and 3(b).

(1) An increase of $a$ gives an increase of both $C_{b}$ and $m_{b}$.

(2) An increase of $b$ gives a decrease of $C_{b}$ and an increase of $m_{b}$

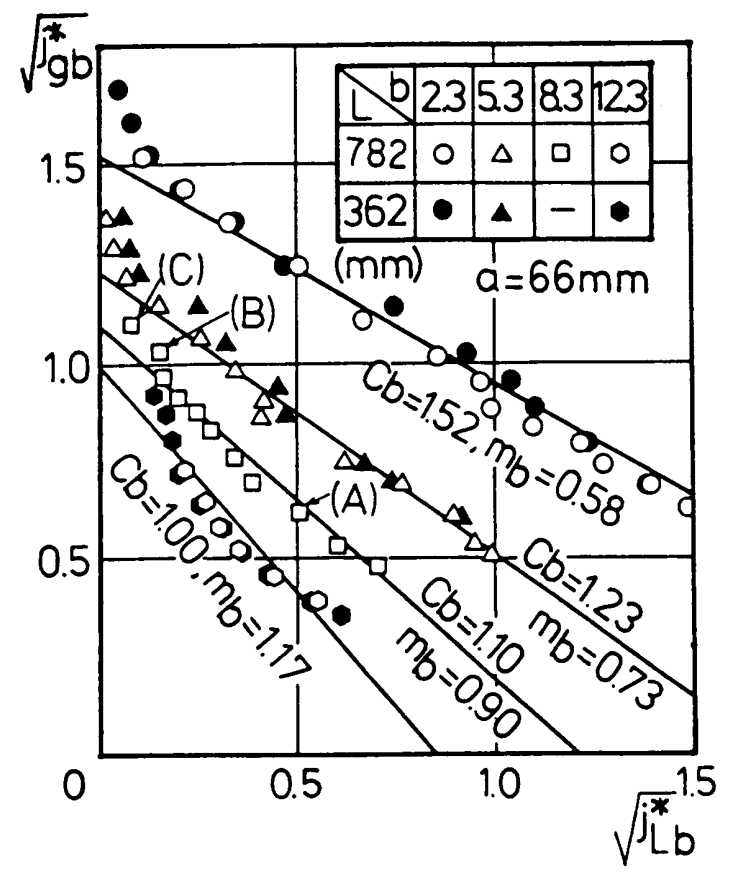

(a) in the case of $a=66 \mathrm{~mm}$

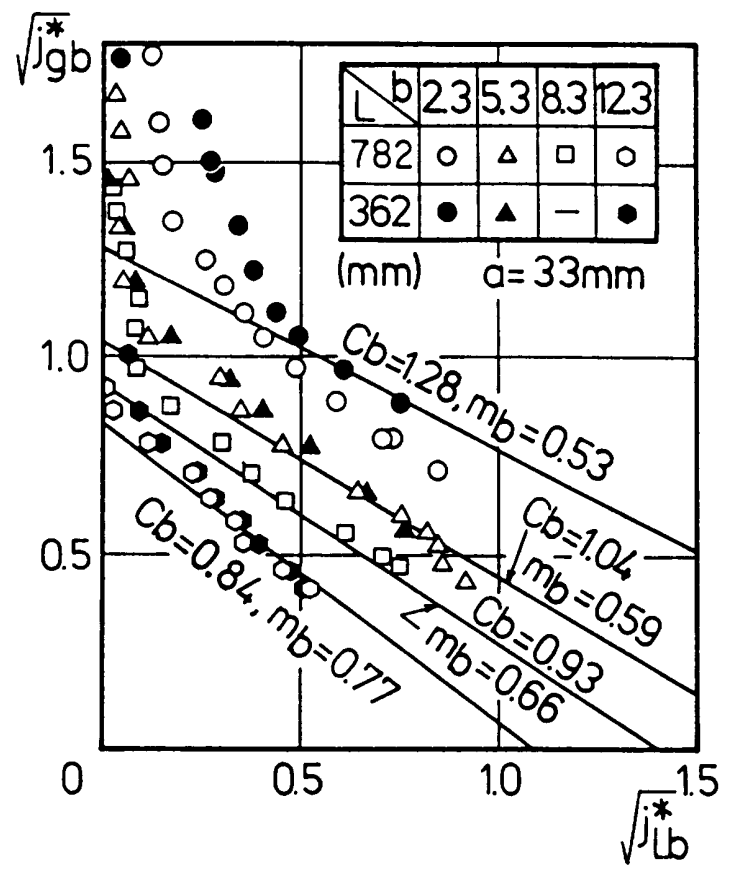

(b) in the case of $a=33 \mathrm{~mm}$

Fig. 3 CCFL experimental results for rectangular channels
Figures 4(a), 4(b) and 4(c) show typical flow patterns for the case of $a=66 \mathrm{~mm}, b=8.3 \mathrm{~mm}$ and $L=$ $782 \mathrm{~mm}$ with $j_{g}=3.1 \mathrm{~m} / \mathrm{s}$ (in Fig. $4(\mathrm{a})$ ). $j_{g}=8.4 \mathrm{~m} / \mathrm{s}$ (in Fig. $4(\mathrm{~b})$ ) and $j_{\theta}=10.1 \mathrm{~m} / \mathrm{s}$ (in Fig. $4(\mathrm{c})$ ).

A wavy liquid film flow is observed on the surface along the shorter side of the cross section of the rectangular test channel in Fig. 4(a). The surface of the longer side of the cross section is wet by a thin liquid film. With an increase of $j_{g}$, dry patches appear on the surface along the longer side of the cross section, as seen in Fig. 4 (b). A further increase of $j_{\theta}$ gives an occurrence of standing waves in the liquid film flowing along the surface of the shorter side of the cross section, as seen in Fig. 4(c). At the top of the standing waves are generated the entrainments of droplets and therefore, once again, the surface along the longer side of the cross section is wet by thin liquid film. The symbols (A) to (C) in Fig. 3 (a ) correspond to the conditions designated by symbols (a) to (c) in Figs. $4(\mathrm{a})$ to $4(\mathrm{c})$.

In the rectangular channels, the experimental results show a significant tendency in which the downward water velocity $j_{L}$ decreases asymptotically to zero with increasing upward gas velocity, $j_{\theta}$, while in circular tubes, the $j_{L}$ decreases linearly with increasing $j_{g}$. It is considered that this is because liquid film flow exists on the surface along the shorter side of the cross section even with high enough $j_{\theta}$, as shown in Fig. 4(c).

Figures 5(a) and 5(b) show the comparisons among typical experimental results obtained in this study, the correlation proposed by Mishima ${ }^{(2)}$ and Eqs. (4) and (5). The experimental data for the conditions of $L=782 \mathrm{~mm}, a=66 \mathrm{~mm}$ and $b=8.3 \mathrm{~mm}$ are

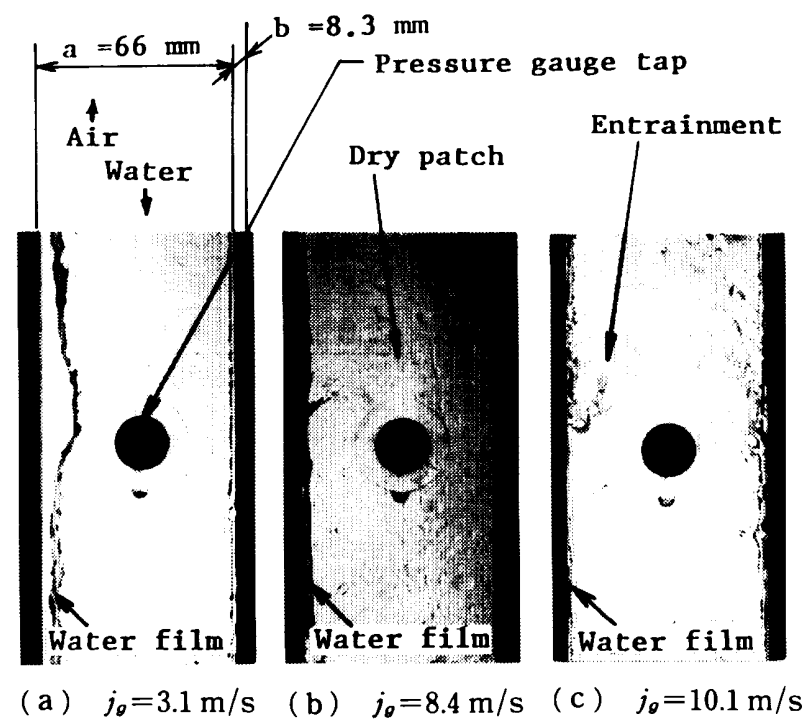

Fig. 4 Flow patterns in the vertical rectangular channels ( $a=66 \mathrm{~mm}, b=8.3 \mathrm{~mm}$ and $L=782 \mathrm{~mm}$ ) 
shown in Fig. 5(a) and those for the conditions of $L$ $=782 \mathrm{~mm}, a=66 \mathrm{~mm}$ and $b=2.3 \mathrm{~mm}$ in Fig. 5 (b).

Figure 5(a) shows a good coincidence between the correlation proposed by Mishima ${ }^{(2)}$ and the experimental results obtained in this study but shows an apparent qualitative and quantitative discrepancy between the experimental results obtained in this study and Eq. ( 4 ) for circular tubes and Eq. ( 5 ) for annular tubes, which were both proposed by the Richter's analysis ${ }^{(5)}$. On the other hand, Fig. 5(b) shows that the correlation proposed by Mishima has a different tendency from that of the experimental results in this study, underestimating $j w_{b}$ much more with decreasing $j_{\theta b}^{*}$ although a rather good coincidence between the two is observed with high $j_{o b}^{*}$. This is considered to be because proper attention is not paid to the effect of channel gap $b$ in the correlation
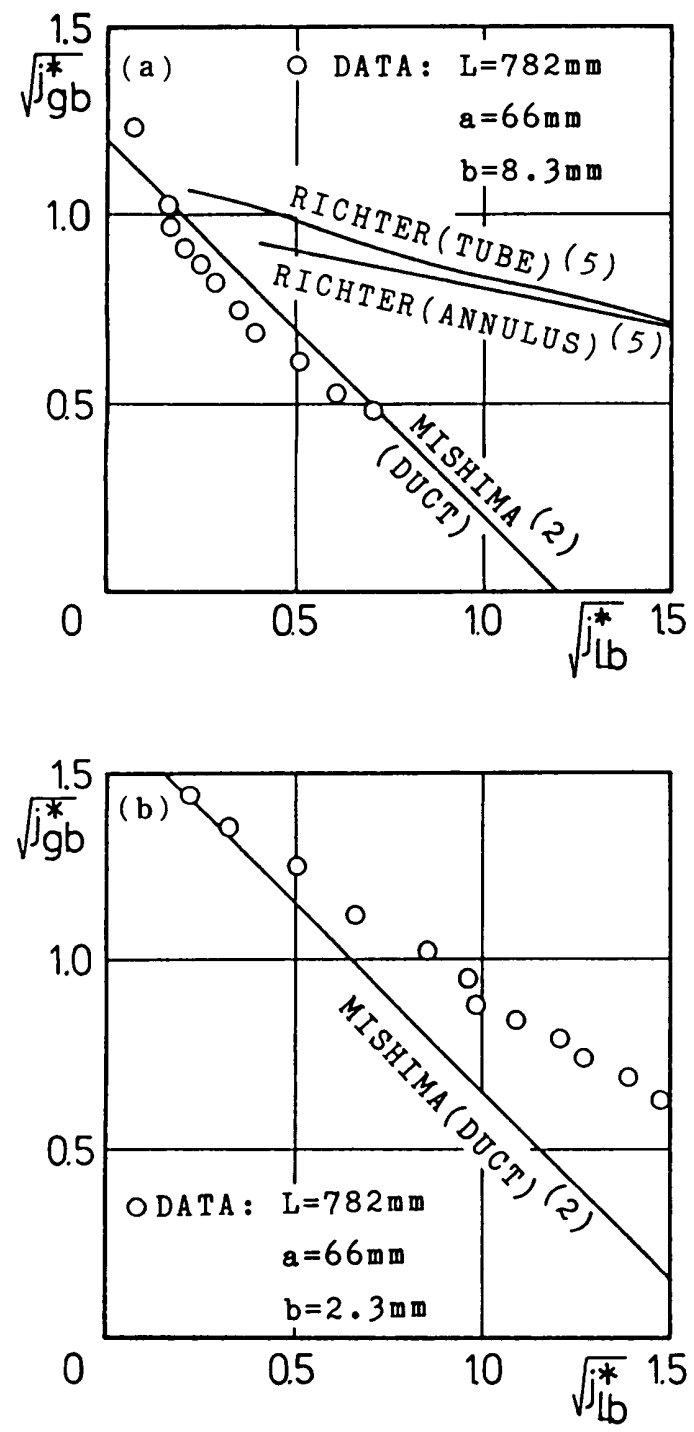

Fig. 5 Comparison of experimental results in this study with previous CCFL correlations proposed by Mishima. It should be pointed out here that Eqs. (4) and (5) do not appear in the range of $\sqrt{j_{\theta b}^{*}}$ vs $\sqrt{j_{L b}^{*}}$ shown in Fig. $5(\mathrm{~b})$.

\section{Introduction of New CCFL Correlation}

From the dimensional analysis, the following key dimensionless parameters other than $j_{o b}^{*}$ and $j_{L_{b}}$ are introduced, which are intimately related to the CCFL in the rectangular vertical channels.

$$
\left.\begin{array}{l}
B o^{*}=\frac{a b\left(\rho_{L}-\rho_{g}\right) g}{\sigma} \\
\frac{b}{a}
\end{array}\right\}
$$

The effects of dimensionless parameters $B o^{*}$ and $\frac{b}{a}$ on $m_{b}$ and $C_{b}$ were first investigated in Fig. 6 . Figure 6 shows the relationship of $\frac{b}{a}$ vs $C_{b}$, where $C_{b}$ has already been determined in Figs. 3(a) and 3(b). The straight line illustrated in Fig. 6 represents the tendency of the experimental data and is given as below.

$$
C_{b}=0.66\left(\frac{b}{a}\right)^{-0.25}
$$

It should be noted here that no systematic effect of $B o^{*}$ on $C_{b}$ appears in Fig. 6.

Figure 7 shows the relationship of $B o^{*}$ vs $m_{b}$, where $m_{b}$ has already been determined in Figs. 3(a) and $3(\mathrm{~b})$. The curve illustrated in Fig. 7 represents

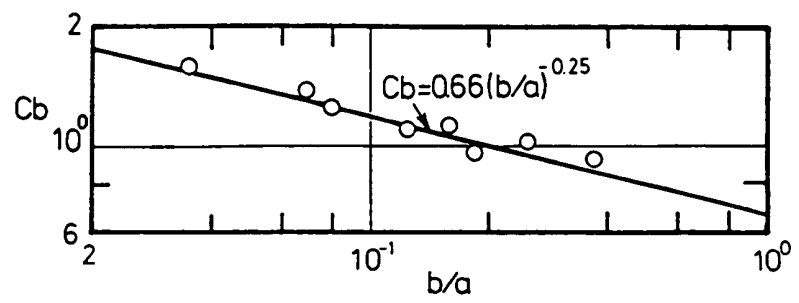

Fig. 6 Relationship of $C_{b}$ vs $b / a$ for rectangular channels

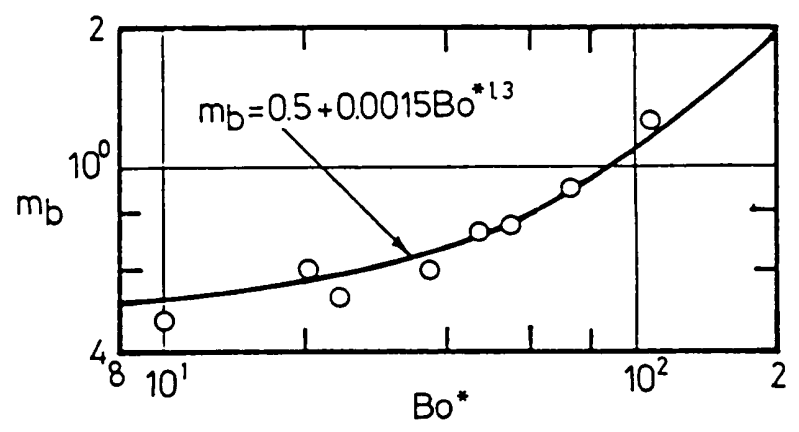

Fig. 7 Relationship of $m_{b}$ vs $B o^{*}$ for rectangular channels 


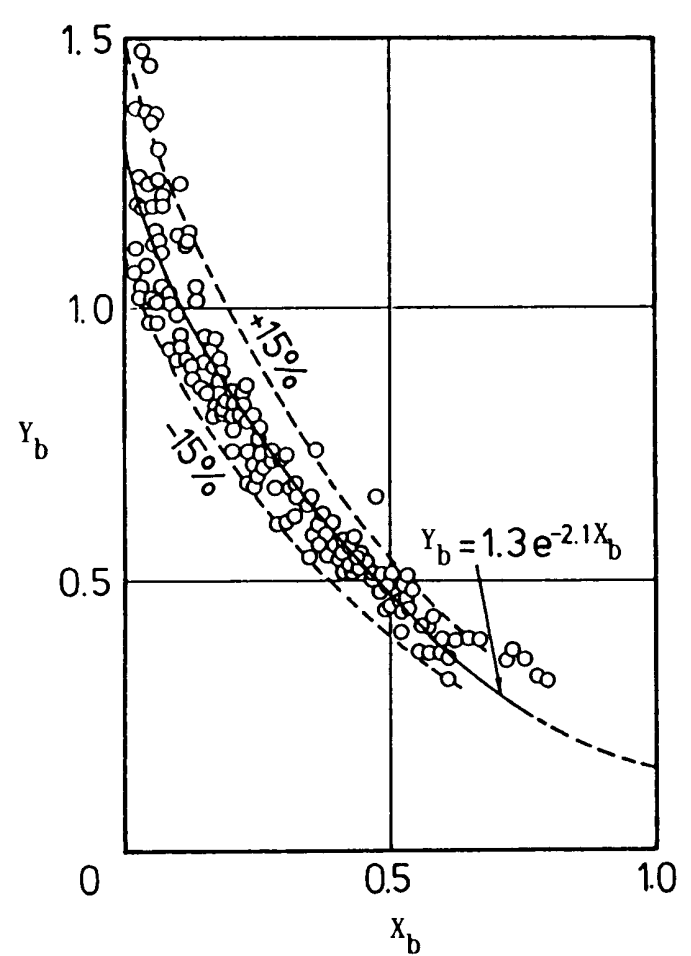

Fig. 8 Experimental data arrangement with new dimensionless parameters, $X_{b}$ and $Y_{b}$, for rectangular channels

the tendency of the experimental data and is given as below.

$$
m_{b}=0.5+0.0015 B o^{* 1.3}
$$

It should also be noted here that no systematic effect of $\frac{b}{a}$ on $m_{b}$ appears.

In order to correlate well the relationship of $j_{b b}^{*}$ vs $j t_{b}$ using $m_{b}$ and $C_{b}$, the following two dimensionless parameters were introduced in this study.

$$
\begin{aligned}
& X_{b}=\left(\frac{m_{b}}{C_{b}}\right) \sqrt{j_{b b}^{*}} \\
& Y_{b}=\left(\frac{1}{C_{b}}\right) \sqrt{j_{g b}^{*}}
\end{aligned}
$$

From Eqs. $(6)$ and $(7)$, the $X_{b}$ and $Y_{b}$ can be rewritten as follows :

$$
\begin{aligned}
& X_{b}=1.52\left(\frac{b}{a}\right)^{0.25}\left(0.5+0.0015 B o^{* 1.3}\right) \sqrt{j_{L b}^{*}} \\
& Y_{b}=1.52\left(\frac{b}{a}\right)^{0.25} \sqrt{j_{a b}^{*} .}
\end{aligned}
$$

Taking these two dimensionless parameters, $X_{b}$ and $Y_{b}$, for the abscissa and the ordinate, respectively, Fig. 8 shows all of the experimental data obtained for the rectangular channels in this study. The solid curve illustrated in Fig. 8 best represents the tendency of the experimental data and is given as follows.

$$
Y_{b}=1.3 \exp \left(-2.1 X_{b}\right)
$$

Equation (13) can predict the experimental data well with the error of $\pm 15 \%$ for the CCFL experimental data obtained in this study by using the dimensionless parameters, $X_{b}$ and $Y_{b}$, as shown in Fig. 8.

\section{Conclusions}

In this study, the CCFL experiments were carried out by systematically varying the magnitude in tube diameter $d$ and tube length $L$ of the vertical circular tubes, and in channel width $a$, channel gap $b$ and channel length $L$ of vertical rectangular channels. As a result, the following conclusions were obtained.

(1) The tendency of the experimental data for vertical circular tubes is well represented by Eq. (1).

(2) An increase of channel gap $b$ gives a significant decrease in $\sqrt{j t_{b}}$ under the condition of the same $\sqrt{j_{a b}^{*}}$ in the case of vertical rectangular channels, while no significant effect of channel length $L$ was observed in this study for the vertical rectangular channel.

(3) In the vertical rectangular channels, it was observed that the $\sqrt{j_{L_{b}}}$ tends to decrease asymptotically to zero with increasing $\sqrt{j_{b b}^{*}}$ because there exists liquid film flowing on the surface along the shorter-side of the cross section even under the condition of high gas velocity.

(4) The CCFL experimental data for vertical rectangular channels can be predicted within the error of $\pm 15 \%$ by Eq. (13), in which the channel width $a$, the channel gap $b$, the dimensionless parameter $B o^{*}$ and the aspect ratio $\frac{b}{a}$ are included as major parameters representing the effects of the configuration of rectangular channels.

\section{References}

(1) Sudo, Y., Miyata, K.. Ikawa, H., Ohgawara, M. and Kaminaga, M., JAERI-M 85-126 (1985) (in Japanese).

(2) Mishima, K., Boillng Burnout at Low Flow Rate and Low Pressure Conditions. Doctoral Dissertation at Kyoto University (1984).

( 3 ) Wallis, G.B., One-Dimensional Two-Phase Flow, McGraw-Hill (1969).

(4) Ueda, T., Gas and Liquid Two-Phase Flow Flow Dynamics and Heat Transfer, Yokendo (1981), p. 90 (in Japanese).

(5) Richter, H.J., Int. J. Multiphase Flow, 7-6 (1981), p. 647.

(6) Sudo, Y., Nucl. Sci. Technol. 21-1 (1984), p. 132.

(7) Sudo, Y. and Ohnuki, A., Trans. JSME, 49-444, B (1982), p. 1658 (in Japanese). 